\title{
Conservative Treatment of Idiopathic Scoliosis: The New Wave of Innovations
}

\author{
Jean Claude de Mauroy* \\ Clinique du Parc Lyon, France \\ *Corresponding author: Jean Claude de Mauroy, Clinique du Parc Lyon, France \\ Submission: 梅 October 06, 2017: Published: 制 November 13, 2017
}

\section{Opinion}

Recent advances in surgery have masked conservative treatments to the point that many patients have no alternative. Below $40^{\circ}$ braces are often prescribed to waiting for surgery. Over $40^{\circ}$, "only surgery can stabilize a scoliosis". There are many reasons for this: the rapidity and effectiveness of surgery, but above all, the lack of knowledge and training for the conservative treatment. Brace prescription is more a matter of local habits and possibilities than a precise objective. However, all braces are not identical and they require a better knowledge of biomechanics. Modern technology combining the very high rigidity of polycarbonate and the hypercorrection of asymmetrical braces can replace plaster casts, but not all orthotists are equipped and trained. 3D correction in sagittal plane and detorsion is possible, but evaluation by systems such as EOS remains limited in some countries. Before definitively blocking the spine under $40^{\circ}$, it is necessary to prescribe the most effective brace adapted to the situation. The effectiveness of the brace should be checked radiologically before payment. For scoliosis more than $40^{\circ}$, modern technologies can be a credible alternative for patients who wish to avoid surgery.

\section{Biomechanical actions can be obtained}

a. Guide growth during night as the support of a tree. Almost all existing rigid braces achieve this goal.

b. Adjust the ligament tensions along the spine. Only the asymmetric braces allow the "rope of the bow" to be stretched. The creep is maximal if the brace is worn full-time like a plaster at the beginning of treatment. 3. Reverse growth asymmetry at the apical vertebra when growth cartilage is still active. Only the hyper-correcting braces worn during the day make it possible to restore a rectangular vertebral body and to break the vicious circle of scoliosis during the growth period. The very high rigidity of the polycarbonate has proved its effectiveness to replace the plaster cast for scoliosis of more than $45^{\circ}$ and thus stabilize without surgery [1].

The association of polycarbonate and asymmetry through a digital cast allows an average hyper correction of $70 \%$ against $33 \%$ for most current braces [2]. The digital cast, the keystone of the hyper-correction is a transposition of the know-how of the correction by Abbott or EDF cast [3]. The corrections are made in the frontal plane and in the sagittal plane successively for the block of pelvis and shoulders, the lumbar block and the thoracic block. The correction in the horizontal plane is obtained automatically by the law of the coupled motions of the spine. The overlay of the 3 blocks produces a torso column or a circled helicoid with a horizontal generating circle, in the opposite direction to the scoliotic spine. However, digital cast requires a quite instantaneous scan because the child cannot maintain the asymmetrical position for more than a few seconds, which excludes all the low-cost scanners currently used.

The very precise protocol of the digital cast facilitates the correction in the 3 planes of the space. In the frontal plane, the correction of $70 \%$ of the Cobb angle that is correlated with the final result is the most important to date and it can be maintained throughout the whole orthopedic treatment. In the sagittal plane, for the first time, pelvic anteversion, lumbar lordosis and thoracic flat back or hyperkyphosis can be corrected separately. In the horizontal plane, thanks to the EOS system, the reality of the detorsion has been proved. The ARTbrace combines geometric and mechanical detorsion. The geometrical detorsion by translation along the vertical axis is obtained by the "pull" under the axillary fold, as when lifting a child by taking him under the arms. Mechanical detorsion via the external surface of the torso is obtained by the "Pringle" effect of the 2 lateral hemi-pieces and the soft contact of the polycarbonate without pad. Bracing scoliosis evolves from plane to solid geometry.

Most of the braces currently prescribed are more than 40 years old, which explains the disaffection of surgeons for the conservative treatment of idiopathic scoliosis. The most recent braces are changing the situation. Below $40^{\circ}$, only noncompliant patients should be treated surgically. Beyond $40^{\circ}$, conservative treatment may be an alternative to stabilize scoliosis without surgery. Technological advances and innovations are making conservative treatment more effective and efficient. The asymmetric braces with hyper-correction and very high rigidity are reducing scoliosis Cobb 
angle by $30 \%$ at the end of treatment. The digital cast in 3 blocks allows a reliable manufacturing and a true three-dimensional correction.

\section{References}

1. Clin J, Aubin C, Sangole A, Labelle H, Parent S (2010) Correlation between immediate in-brace correction and biomechanical effectiveness of brace treatment in adolescent idiopathic scoliosis. Spine (Phila Pa 1976) 35(18): 1706-1713.
2. Negrini S, Negrini F, Fusco C, Zaina F (2011) Idiopathic scoliosis patients with curves more than 45 Cobb degrees refusing surgery can be effectively treated through bracing with curve improvements. Spine J 11(5): 369-380.

3. de Mauroy JC, Journe A, Gagaliano F, Lecante C, Barral F, et al. (2015) The new Lyon ARTbrace versus the historical Lyon brace: a prospective case series of 148 consecutive scoliosis with short time results after 1 year compared with a historical retrospective case series of 100 consecutive scoliosis; SOSORT award 2015 winner. Scoliosis 10: p. 26. 